\title{
Photoelektrochemischer Sarkosin-Nachweis auf Grundlage von CdSe/ZnS Quantum Dots und Sarkosinoxidase
}

\author{
Marc Riedel, Gero Göbel, Fred Lisdat
}

Biosystemtechnik, TH Wildau, Bahnhofstrasse 1, 15745 Wildau, Germany

flisdat@th-wildau.de

\begin{abstract}
Zusammenfassung
Diese Arbeit beschäfftigt sich mit dem photoelektrochemischen Sarkosin-Nachweis auf Grundlage von CdSe/ZnS Quantum Dots. Dafür werden Elektroden mit Quantum Dots modifiziert und der sauerstoffabhängige Photostrom unter Beleuchtung ausgewertet. Die Photostromgenerierung beruht auf der Ladungsträgererzeugung in den angeregten QDs und Elektronentransferreaktionen zu Elektoden bzw. Donor/Akzeptorsubstanzen. Mit dem Enzym Sarkosinoxidase (SOX) kann der Photostrom in Abhängigkeit von der Sarkosinkonzentration durch die biokatalytische Reduktion des Sauerstoffs unterdrückt werden. Die Immobilisierung des Enzyms wird durch die abwechselnde Assemblierung mit dem Polyelektrolyten Polyallylaminhydrochlorid (PAH) über elektrostatische Wechselwirkungen realisiert. Auf diese Weise können Multischichtsysteme mit bis zu 6 (SOX/PAH)Schichten aufgebaut und mittels SPR verifiziert werden. Die Schichtanzahl hat einen signifikanten Einfluss auf die Menge an assembliertem Enzym und die Sensitivität des Sensors.
\end{abstract}

Schlüsselwörter: Elektrochemie, Quantum Dots, Enzyme, Photoelektrochemie, Sensoren

\section{Einleitung}

Quantum Dots (QDs) sind kolloidale Halbleiternanopartikel, welche bereits in zahlreichen Disziplinen wegen der interessanten optischen Eigenschaften Anwendung finden [1,2]. Es werden für die Synthese hauptsächlich II-IV-Halbleiter (z.B. CdSe, CdS, CdTe, ZnS) verwendet. QDs werden häufig nach dem Kern-Schale-Prinzip mit einem zweiten Halbleiter (z.B. CdSe/ZnS) überzogen um den Kern vor Photobleaching zu schützen und die Quantenausbeute zu steigern. Aufgrund der Halbleitereigenschaften können in den QDs durch die Bestrahlung mit Licht ausreichender Energie Elektronen-Loch-Paare generiert werden, bei denen ein Elektron in das Leitungsband übergeht und ein Defektelektron im Valenzband verbleibt. Die benötigte Anregungsenergie ist abhängig von der Bandlücke zwischen dem Valenz- und Leitungsband. Die Energie der Bandlücke korreliert dabei invers mit dem Durchmesser der QDs. Das heißt je kleiner die QDs sind, umso mehr Energie ist nötig um einen Elektronsprung über die Bandlücke in das Leitungsband zu induzieren. Eine Relaxation der angeregten Elektronen in den
Grundzustand führt zur Emission von Licht (Fluoreszenz). Die Energie des ausgestrahlten Lichtes ist abhängig vom Durchmesser der QDs und ermöglicht eine größen-gesteuerte, parallele Multiplexanalyse.

Neben der optischen Nutzung werden QDs verstärkt für den Aufbau von elektrochemischen Sensoren als lichtsensitives Element eingesetzt [3-6]. Durch die Fixierung von QDs auf Elektroden können Licht-induzierte Ladungsträger abgefangen und in ein Stromsignal umgewandelt werden. Wie in Abbildung 1 dargestellt kann abhängig vom Potential ein anodischer oder kathodischer Photostrom detektiert werden. Der anodische Photostrom wird durch eine Elektronentransferreaktion von einem Donor in Lösung über die QDs zur Elektrode ausgelöst. Hingegen wird der kathodische Photostrom durch den Übergang der Elektronen von der Elektrode über die QDs zu einem Akzeptor in der Lösung generiert. In Abwesenheit von Licht verhalten sich die QDs isolierend und es ist kein Elektronentransfer möglich. 

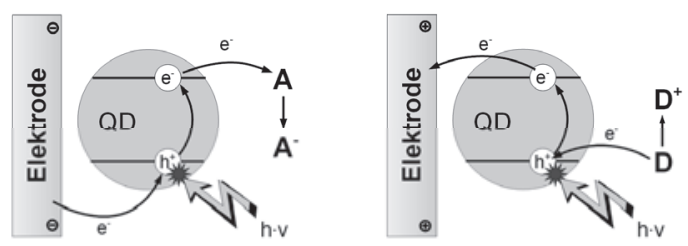

Abb. 1 Elektronentransferschritte an einer $Q D$ modifizierten Elektrode nach der Beleuchtung. Links: Kathodischer Photostrom. Rechts: Anodischer Phtostrom.

Um Quantum Dots auf der Elektrode zu fixieren und eine bessere Wechselwirkung mit biologischen Molekülen zu gewährleisten, können die Oberflächenliganden der QDs ausgetauscht werden. Dabei werden die oft unpolaren Oberflächenliganden (z.B. Trioctylphosphine) der QDs nach der Synthese durch Liganden mit funktionellen Gruppen ersetzt. Dithiolverbindungen werden dabei häufig für die Immobilisierung auf Goldoberflächen über Chemisorption verwendet.

Quantum Dot Elektroden können mit verschiedenen redoxaktiven Molekülen kombiniert werden. So konnte gezeigt werden, dass Kofaktoren von Enzymen wie z.B. NADH an QD-Elektroden in Abhängigkeit vom Potential über eine Photostromzunahme detektiert werden können [7]. Dies ermöglicht den Zugang zum Nachweis von Substraten von $\mathrm{NADH}$-abhängigen DehydrogenaseReaktionen. Außerdem können auch Bedingungen gefunden werden um Redoxproteine in direkten, elektrochemischen Kontakt mit den QDs zu bringen und so Interaktionspartner des Proteins über Photostrommessungen nachzuweisen [8]. Dieses Prinzip kann verwendet werden um Signalketten mit Cytochrom c und Enzymen aufzubauen. Zum Beispiel werden QDElektroden mit Cytochrom c und Laktatdehydrogenase kombiniert um Laktat über einen Anstieg im anodischen Photostrom nachzuweisen. Der Austausch des enzymatischen Systems durch Nitratreduktase führt hingegen in Anwesenheit von Nitrat zu einem steigenden kathodischen Photostrom[9].

Des Weiteren hat sich gezeigt, dass der kathodische Photostrom abhängig von der Sauerstoffkonzentration in Lösung ist [10]. Sauerstoff fungiert dabei als Elektronenakzeptor der angeregten QDs. Dieser Effekt wurde in dieser Studie verwendet um die Kombination von QD-Elektroden mit einer spezifischen biochemischen Reaktion für die sensorische Anwendung zu untersuchen.

\section{Einfluss von Sauerstoff auf den Photostrom}

Für die Entwicklung des Sensors wurden CdSe/ZnS Quantum Dots mit einem Durchmesser von 3,1nm mit Hilfe eines Dithiollinkers auf Goldelektroden immobilisiert. Der Einfluss von Sauerstoff auf den kathodischen Photostrom der QDs wird in luftgesättigtem und in Argon gespültem Puffer untersucht. Dazu wird Licht im 10 Sekundentakt ein- und ausgeschaltet. Es kann beobachtet werden, dass der Photostrom nach 6 - 8 Lichtimpulsen konstant wird. Voltammetrische Messungen des sauerstoffabhängigen Photostroms zeigen eine klare Abhängigkeit vom $\mathrm{pH}$-Wert und vom angelegten Potential. Ein Potential kleiner -0,3V vs. $\mathrm{Ag} / \mathrm{AgCl}$ und ein neutraler $\mathrm{pH}-$ Wert sind die Voraussetzungen für eine effektive Photostromgenerierung. Der kathodische Photostrom ist bei einem Potential von $-350 \mathrm{mV}$ vs. $\mathrm{Ag} / \mathrm{AgCl}$ und $\mathrm{pH} \mathrm{7,6}$ in $\mathrm{Abwesenheit}$ von Sauerstoff 50 - $60 \%$ kleiner als in luftgesättigter Lösung (siehe Abbildung 2). Das bedeutet, dass unter den experimentellen Bedingungen Sauerstoff als Elektronenakzeptor dient und eine vollständige Rekombination der Licht-induzierten Elektronen-Loch-Paare in den QDs verhindert, was sich in einem konstanten Photostrom äußert. Dieser erweist sich als linear abhängig von der Sauerstoffkonzentration in der Lösung.

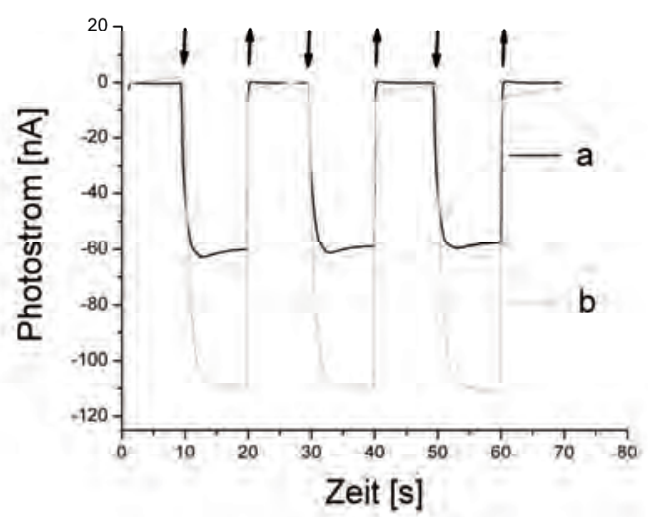

Abb.2 Photostrommessungen einer CdSe/ZnS QDmodifizierten Elektrode in Argon-gespülten (a) und Luft-gesättigten Puffer (b) [100mM HEPES pH 7,6; $350 \mathrm{mV}$ vs. Ag/AgCl; die Pfeile kennzeichnen das anund ausschalten der Lichtquelle]

Die Sauerstoffabhängigkeit bietet die Basis für die Kombination von QDs mit dem Enzym Sarkosinoxidase (SOX) für den Nachweis von Sarkosin. Wie in Abbildung 2 dargestellt führt die kompetitive Situation zwischen den QDs und dem Enzym um den Sauerstoff zu einer Signalveränderung. 


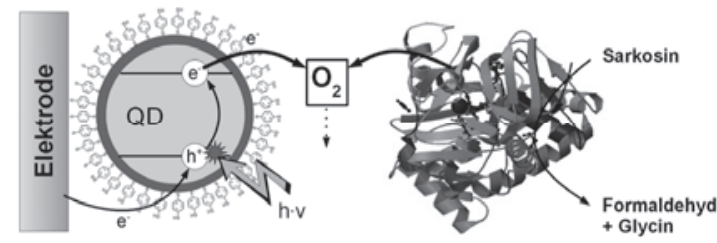

Abb.3 Sensorprinzip einer QD-Elektrode in Kombination mit dem Enzym Sarkosinoxidase zum Sarkosinnachweis. Die angeregten QDs und das Enzym konkurrieren um den verfügbaren Sauerstoff in Anwesenheit von Sarkosin.

\section{Untersuchung der SOX-QD-Elektrode}

Der Aufbau einer Sensorelektrode erfolgt durch eine schichtweise Immobilisierung von Sarkosinoxidase auf den QDs. Dies kann durch abwechselnde Assemblierung von SOX und dem Polyelektrolyten Polyallylaminhydrochlorid (PAH) erreicht werden. Dieses Prinzip basiert auf den elektrostatischen Wechselwirkungen zwischen dem negativ geladenen Enzym und dem positiv geladenen Polyelektrolyten. Mit Hilfe von SPR Untersuchungen können die Bedingungen für die Bildung dieses Multischichtsystems evaluiert werden.

Eine Fixierung der SOX auf der QD-Elektrode führt in Anwesenheit von Sarkosin zu einer Photostromunterdrückung in Abhängigkeit von der Substratkonzentration. Die Sensitivität des Sensors kann über die Menge an abgeschiedenem Enzym durch die Variation der Anzahl an [SOX/PAH]-Schichten auf den QD-Elektroden eingestellt werden. Wie in Abbildung 4 dargestellt kann für Schichtsysteme im Bereich von 2 - 6 Schichten eine Photostromunterdrückung in Anwesenheit von Sarkosin festgestellt werden, wobei die Signalantwort mit der Anzahl an Sarkosinoxidaseschichten steigt. Die Sensitivität des [SOX/PAH] $]_{6}-\mathrm{QD}$ Sensors liegt bei $0,041 \% / \mu \mathrm{M}$ Sarkosin mit einem linearen Bereich zwischen $100 \mu \mathrm{M}$ und $1 \mathrm{mM}$. Der dynamische Messbereich verläuft bis $30 \mathrm{mM}$ Sarkosin. Die Untersuchungen zeigen, dass Sarkosin in das ganze Schichtsystem diffundieren kann und Enzyme in der Nähe der Elektrode auch an der Generation des enzymatischen Signals beteiligt sind. Unter Substratsättigung kann der Photostrom zu 60\% unterdrückt werden. Dies entspricht dem sauerstoffabhängigen Teil des Photostroms. Es kann also geschlussfolgert werden, dass mit dem 6 Schichtsystem genügend SOX assembliert werden kann um die komplette Sauerstoffsensitivität der QD-Elektrode auszunutzen. Nach der Messung von verschiedenen Substratkonzentrationen kann der Sensor in Puffer regeneriert werden und ist für weitere Messungen bereit.

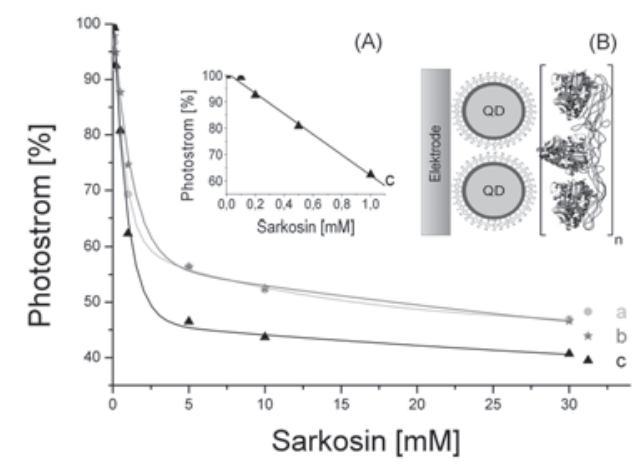

Abb. 4 Relative Photostromänderung der $Q D$ modifizierten Elektroden mit Sarkosinoxidase in Abhängigkeit von der Sarkosinkonzentration von 0 $30 \mathrm{mM}$ und $0-1 \mathrm{mM}$ (A) für das a) (SOX/PAH) $)_{2}$, b) $(\mathrm{SOX} / \mathrm{PAH})_{4}$ und c) (SOX/PAH) 6 Schichtsystem. (B) Schematische Darstellung der (SOX/PAH)Assemblierung auf einer QD-Elektrode.

Die Photostrommessung von Sarkosin an einer QD-Elektrode ohne Enzym führt zu keiner Signalabnahme. Daraus kann geschlussfolgert werden, dass es zu keiner direkten Umwandlung von Sarkosin an den beleuchteten QDs kommt und die Photostromabnahme allein auf den enzymatischen Sauerstoffverbrauch vor der Elektrode zurückzuführen ist. Eine Interaktion der Produkte der Enzymreaktion mit den QDs kann ebenfalls nicht beobachtet werden.

Das hier vorgestellte Sensorprinzip auf Basis von immobilisierten QDs besitzt großes Potential für die parallele Analyse von verschiedenen Enzymsubstraten, wenn die jeweiligen Biokatalysatoren separat auf der Oberfläche immobilisiert werden und eine räumlich aufgelöste Bestrahlung der einzelnen Spots verwendet wird.

\section{Zusammenfassung}

In dieser Arbeit wurde ein auf CdSe/ZnS QDs und SOX basierender Photoelektrochemischer Sensor für die Bestimmung der Sarkosinkonzentation entwickelt. Zunächst kann die Sauerstoffabhängigkeit des kathodischen Photostroms während der Beleuchtung gezeigt werden. Des Weiteren wird mit der "layer-by-layer" Technik eine effiziente Methode zur Steigerung der Enzymkonzentration vor der Elektrode eingesetzt. Die Kombination von SOX mit der QD-Elektrode führt in Anwesenheit von Sarkosin zu einer Photostromunterdrückung aufgrund der Konkurrenzsituation um den Sauerstoff in der Lösung. Der lineare 
Messbereich liegt zwischen $100 \mu \mathrm{M}$ und $1 \mathrm{mM}$ und die Sensitivität ist abhängig von der Anzahl an Enzymschichten. Die Studie verdeutlicht das Potential der Kombination von QDs mit anderen
Sauerstoff verbrauchenden Enzymen zur Etablierung von Licht-gesteuerten

\section{Literatur}

[1] W.J. Parak, T. Pellegrino, C. Plank,

Nanotechnology 16, R5-R25 (2005); doi: 10.1088/0957-4484/16/2/R01

[2] C. Wang, X. Gao, X.G. Su, Analytical And Bioanalytical Chemistry 397, 1397-1415 (2011); doi: $10.1234 / \mathrm{s} 10000$

[3] F.Lisdat, D. Schäfer, A. Kapp, Analytical Bioanalytical Chemistry 405, 3739-3752 (2013); doi: 10.1007/s00216-013-6789-1

[4] L. Tang, Y Zhu, X. Yang, J. Sun, C. Li, Biosensors \& Bioelectronics 24, 319-323 (2008); doi: 10.1016/j.bios.2008.03.043

[5] S.G. Hickey, D.J. Riley, Electrochimica Acta 45, 3277-3282 (2000); doi: 10.1016/S00134686(00)00431-X

[6] M.L. Curri, A. Agostiano, G. Leo, A. Mallardi, P. Cosma, M. Della Monica, Materials of Science and Engineering C 22, 449-452 (2002); doi: 10.1016/S0928-4931(02)00191-1

[7] K. Schubert, W. Khalis, Z. Yue, W.J. Parak, F. Lisdat, Langmuir 26, 1395-1400 (2010); doi: 10.1021/la902499e

[8] C. Stoll, S. Kudera, W.J. Parak, F. Lisdat, Small 2, 741-743 (2006); doi: 10.10021/smll.200500441

[9] E. Katz, M. Zayazs, I. Willner, F. Lisdat, Chemiical Communication 13, 1395-1397 (2006); doi: 10.1039/B517332A

[10] J. Tanne, D. Schäfer, W. Khalis, W.J. Parak, F. Lisdat, Analytical Chemistry 83, 7778-7785 (2011); doi: 10.10021/ac201329u 